Miguel Ángel Fernández-Barrera ${ }^{1}$, Teresita de Jesús Saucedo-Molina ${ }^{2}$, Rogelio José Scougall-Vilchis³ ${ }^{3}$ María de Lourdes MárquezCorona $^{3}$, Carlo Eduardo Medina-Solís ${ }^{1,3}$, Gerardo Maupomé $e^{4,5}$

\title{
Comparison of Two Types of Pit and Fissure Sealants in Reducing the Incidence of Dental Caries Using a Split-Mouth
} Design

\section{Usporedba dviju vrsta smola za pečaćenje fisura i jamica u smanjenju incidencije zubnog karijesa primjenom dizajna podijeljenih usta}

\author{
${ }^{1}$ Academic Area of Dentistry of Health Sciences Institute at Autonomous University of Hidalgo State. Pachuca, Mexico. \\ Akademsko područje za stomatologiju Instituta zdravstvenih znanosti pri Autonomnom sveučilištu države Hidalgo, Pachuca, Meksiko \\ 2 Academic Area of Nutrition of Health Sciences Institute at Autonomous University of Hidalgo State. Pachuca, Mexico. \\ Akademsko područje za prehranu Instituta za zdravstvene znanosti pri Autonomnom sveučilištu države Hidalgo, Pachuca, Meksiko \\ ${ }^{3}$ Advanced Studies and Research Centre in Dentistry “Dr. Keisaburo Miyata” of School of Dentistry at Autonomous University of the State of \\ Mexico. Toluca, Mexico. \\ Centar za napredne studije i istraživanja u stomatologiji Dr. Keisaburo Miyata sa Stomatološkog fakulteta pri Autonomnom sveučilištu države \\ Meksiko, Toluca, Meksiko \\ ${ }^{4}$ Richard M. Fairbanks School of Public Health, Indiana University/Purdue University. Indianapolis, USA. \\ Richard M. Fairbanks School of Public Health, Sveučilište Indiana/Sveučilište Purdue. Indianapolis, SAD \\ ${ }^{5}$ Indiana University Network Science Institute, Bloomington, USA. \\ Institut za znanost o mrežama Sveučilišta Indiana, Bloomington, SAD
}

\section{Abstract}

Objectives: To evaluate the effectiveness of two pit and fissure sealants (PFS) in reducing the incidence of dental caries in schoolchildren. Material and Methods: A randomized split-mouth experimental design was used in a sample of 140 subjects assigned to two groups. The sealants used were Clinpro $03 \mathrm{M}$ and BeautiSealantCShofu placed in first permanent molars (FPMs). Each sealant was compared to molars in the controls to determine effectiveness over a period of 6 months. The study had a $12.9 \%$ loss to follow-up. No statistically significant differences $(p>0.05)$ were observed for sex, age, baseline dmft, or type of sealants. Nonparametric tests were used for statistical analysis. Results: Average dmft index at baseline was 4.10 \pm 3.16 . Lower incidence of caries was observed in FPMs with pit and fissure sealants $(p<0.01)$, regardless of the type used. When sealants remained intact there was a lower caries incidence compared to sealants partially or completely missing - but differences were only significant for FPM 16. Caries incidence was higher for BeautiSealant sealed teeth than for Clinpro's, but only statistically significant in FPMs 16, 36 and $46(p<0.05)$. Caries incidence was higher in those cases with a higher baseline dmft but it only reached statistical significance in FPMs 26 and 36. Relative risks for dental caries were lower in sealed teeth $(p<0.01)$. Conclusions: Pit and fissures sealants are an effective preventive treatment to reduce caries during a 6-month followup in schoolchildren 6 to 8 years of age, regardless of the type of sealant used. The sealant brand that showed greater effectiveness in terms of prevention and retention was Clinpro@3M.
Received: July 6, 2020

Accepted: February 24, 2021

Address for correspondence MSc. Carlo Eduardo Medina-Solís Avenida Álamo \# 204,

Fraccionamiento Paseo de los Solares.

CP. 42113. Pachuca de Soto, Hidalgo., Mexico.

cemedinas@yahoo.com

MeSH terms: Pit and Fissure Sealants; Dental Caries; Child

Author keywords: Pit and Fissure Sealants; Oral Health; Caries

\section{Introduction}

According to the Global Burden of Disease in 2010, untreated caries in permanent and primary teeth represented the first and tenth most prevalent condition, respectively (1). In Latin America, oral diseases constitute a public health problem (2). Specifically, in Mexico six-year olds have caries on their primary teeth, with caries index ranging from 0.73 to 5.35 (decayed, missing and filled primary teeth (dmft index)), and caries prevalence is between $26.3 \%$ and $77.5 \%$.
Uvod

Prema istraživanju globalnog opterećenja bolesti iz 2010., neliječeni karijes na trajnim i mliječnim zubima bio je prvo, odnosno deseto najzastupljenije stanje (1). U Latinskoj Americi oralne su bolesti javnozdravstveni problem (2). Točnije, u Meksiku je šestogodišnjacima na mliječnim zubima zabilježen indeks karijesa u rasponu od 0,73 do 5,35 (mliječni zubi $s$ karijesom, izvađeni mliječni zubi i mliječni zubi s ispunom - DMFT indeks) i prevalencija karijesa između 26,3 i 77,5 
For permanent teeth, dental caries index among 12-year olds ranged from 0.52 to 3.67 (Decayed, Missing and Filled Permanent Teeth (DMFT index)) and caries prevalence ranged between $30.7 \%$ and $79.2 \%$ (3). For 15 -year-olds, the average DMFT ranged between 1.12 and 5.31, and prevalence between $37.6 \%$ and $88.6 \%$ (3). The results of the studies conducted in developing countries revealed that more than $80 \%$ of caries lesions in primary and permanent teeth required restorative treatment. The high prevalence and treatment needs represent an economic burden for both public health systems and households, with the latter often paying out-of-pocket expenses for private care $(4,5)$.

First permanent molars (FPMs) are the earliest teeth to erupt in the secondary dentition (6). The occlusal morphology of pit and fissures is an important reason for dental caries at this surface. While diverse restorative materials can be used to restore occlusal cavities (7), pit and fissure sealants are highly effective preventive interventions whereby a lowviscosity resin is placed in pits and fissures (8-10). In Mexico, less than $2.5 \%$ of children and adolescents aged 6 - 12-years had sealants of any type (11).

The present study set out to ascertain the effectiveness of sealants in general in a group of community-dwelling children in terms of caries prevention performance in first permanent molars (FPM). Secondly, we compared the performance of two commonly used sealants: ClinPro@3M and BeautiSealant@Shofu pit and fissure sealants.

\section{Material and methods}

\section{Design}

We conducted a randomized controlled split-mouth clinical trial in elementary school children in Hidalgo, Mexico. Figure 1 shows the methodological design. Our design included the conventional Clinpro sealant (Clinpro@3M, Saint Paul, MN., USA) and one sealant based on Giomer technology (BeautiSealant@Shofu, Kyoto, Japan). Clinpro@3M is a resin-based product that requires an etching adhesive system (12). BeautiSealant@Shofu sealant uses Giomer technology with an adhesive system that does not require phosphoric acid (13). Different technologies may affect various aspects of performance (e.g., pit penetration (14), retention (15) and microfiltration (16), which was not individually addressed in the present research.

Our research protocol was approved by the Ethics Committee of the Health Sciences Institute at the Autonomous University of the State of Hidalgo (Cinv/o/032/2016).

\section{Sealant application}

Dental prophylaxis with fluoride-free abrasive paste and rubber cup was carried out at low speed. Relative isolation was attained with cotton rolls. A conventional adhesive system was followed with the Clinpro sealant; that is, the enamel surface was etched for 20 seconds, and after rinsing with plenty of water, the sealant was placed with applicator tips (3MESPE). For the BeautiSealant sealant, first conditioning was attained with air gently applied for 5 seconds, followed by placing the sealant (Shofu). Both products were cured
$\%$. Za trajne zube indeks karijesa među 12-godišnjacima bio je od 0,52 do 3,67 (trajni zubi s karijesom, izvađeni mliječni zubi i mliječni zubi s ispunom - DMFT indeks), a prevalencija karijesa kretala se između 30,7 i 79,2 \% (3). Za 15-godišnjake je prosječni DMFT indeks iznosio između 1,12 i 5,31, a prevalencija između 37,6 i 88,6 \% (3). Istraživanja provedena u zemljama u razvoju pokazala su da više od $80 \% \mathrm{ka}$ rijesnih lezija na mliječnim i trajnim zubima zahtijeva restaurativno liječenje. Velika prevalencija i potreba za liječenjem financijsko su opterećenje i za javnozdravstveni sustav i za kućanstva, pri čemu kućanstva često plaćaju privatno liječenje iz vlastita džepa $(4,5)$.

Prvi trajni kutnjaci prvi su zubi koji niknu u trajnoj denticiji (6). Okluzalna morfologija fisura i jamica na tom je dijelu uzrok nastanka zubnog karijesa. Iako se različiti materijali mogu upotrijebiti za restauraciju okluzalnih kaviteta (7), smole za pečaćenje fisura i jamica vrlo su učinkovite u prevenciji, pri čemu se u fisure i jamice aplicira smola niske viskoznosti $(8-10)$. U Meksiku je kod manje od 2,5 \% djece i adolescenata između 6 i 12 godina primijenjena smola za pečaćenje bilo koje vrste (11).

Cilj ovog istraživanja bio je općenito analizirati učinkovitost smola za pečaćenje fisura u skupini djece u smislu prevencije karijesa na prvim trajnim kutnjacima. Kao drugo, usporedili smo performanse dviju često korištenih smola za pečaćenje fisura i jamica - $\mathrm{ClinPro}^{\odot} 3 \mathrm{M}$ i BeautiSealant ${ }^{\oplus}$ Shofu.

\section{Materijali i metode}

\section{Studijski dizajn}

Proveli smo randomizirano kontrolirano kliničko istraživanje s podijeljenim ustima kod djece osnovnoškolske dobi u Hidalgu u Meksiku. Na slici 1. je metodološki dizajn. Upotrijebljeni materijali za pečaćenje bili su Clinpro (Clinpro ${ }^{\odot}$ 3M, Saint Paul, MN., SAD) i jedan temeljen na giomernoj tehnologiji (BeautiSealant ${ }^{\oplus}$ Shofu, Kyoto, Japan). Clinpro ${ }^{\odot}$ $3 \mathrm{M}$ proizvod je na bazi smole koji zahtijeva adhezivni sustav za jetkanje (12). BeautiSealant ${ }^{\odot}$ Shofu smola je na bazi giomerne tehnologije $s$ adhezivnim sustavom koji ne zahtijeva ortofosfornu kiselinu (13). Različite tehnologije mogu utjecati na različite aspekte izvedbe, na primjer, na prodiranje u fisure (14), retenciju (15) i mikrofiltraciju (16), što nije obrađeno pojedinačno u ovom istraživanju.

Naš protokol za istraživanje odobrio je Etički odbor Instituta za zdravstvene znanosti pri Autonomnom sveučilištu države Hidalgo (Cinv/o/032/2016).

\section{Aplikacija smole za pečaćenje}

Najprije je provedena profilaksa abrazivnom pastom bez fluorida i gumenim polirerom pri maloj brzini. Razmjerno suho radno polje postignuto je svitcima staničevine. Nakon konvencionalnog adhezivnog sustava aplicirana je smola Clinpro, to jest površina cakline jetkana je 20 sekunda, a nakon ispiranja s obilno vode smola je nanesena vrhom aplikatora (3M ESPE). Za smolu BeautiSealant prvo je kondicioniranje postignuto ispuhivanjem zrakom tijekom 5 sekunda, nakon čega je slijedila aplikacija smole (Shofu). Oba proizvo- 


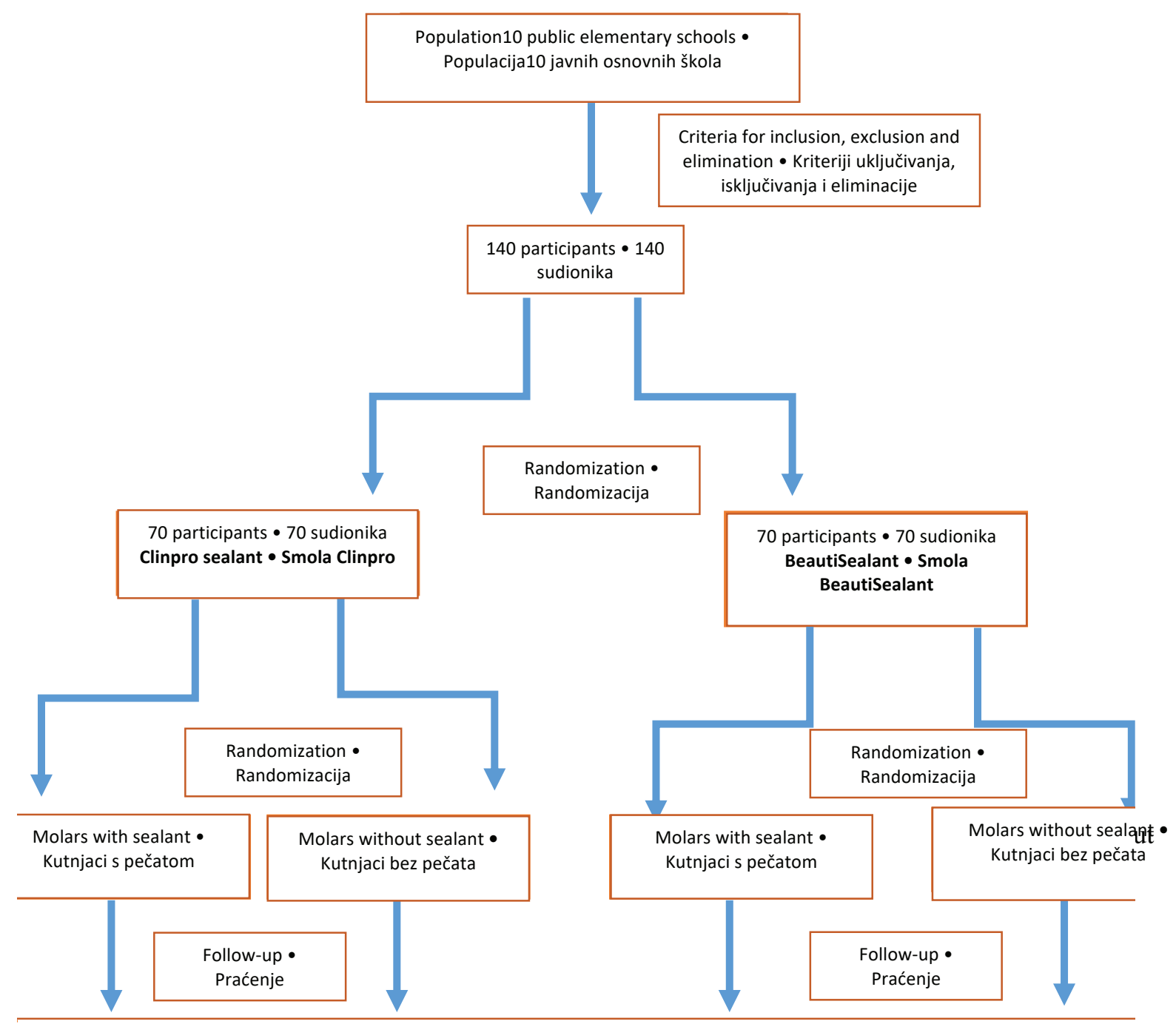

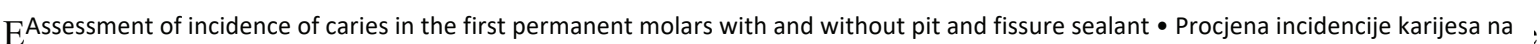
prvim trajnim kutnjacima sa smolom za pečaćenje fisura i jamica bez smole

Figure 1 Flowchart of methodological design of the study

Slika 1. Dijagram metodologije istraživanja

with a LED lamp for 20 seconds. We followed the manufacturers' instructions.

\section{Study sample and participant selection}

A formula for proportional difference was used to calculate the sample size. The prevalence of caries in the FPMs with and without sealants (30\% and 60\%, respectively) was based on a p-value of 0.05 and $90 \%$ power. We added $10 \%$ loss to follow-up calculations, leading to 70 participants per sealant.

We selected the participants randomly from ten public elementary schools after holding a meeting with heads of households in each school, before asking for their signed informed consent. Inclusion criteria were (1) having four fully erupted and sound FPMs; (2) being enrolled in one of the ten selected schools; (3) being between six and eight years old; (4) having no fixed orthodontic appliances; and (5 the child assenting to go through dental examination. da polimerizirana su LED svjetiljkom 20 sekunda. Slijedili smo upute proizvođača.

\section{Uzorak i odabir ispitanika}

$\mathrm{Za}$ izračunavanje veličine uzorka korištena je formula proporcionalne razlike. Prevalencija karijesa na prvim trajnim kutnjacima sa smolom za pečaćenje i bez nje (30 \%, odnosno $60 \%$ ) temeljila se na p-vrijednosti od 0,05 do $90 \%$ snage. Proračunima smo dodali gubitak od $10 \%$ ispitanika tijekom praćenja, što je rezultiralo $s$ ukupno 70 sudionika po smoli za pečaćenje.

Sudionike smo odabrali nasumično iz deset javnih osnovnih škola nakon sastanka s ravnateljima, prije nego što smo zatražili njihov potpisani informirani pristanak. Kriteriji za uključivanje bili su (1) četiri potpuno eruptirana i zdrava prva traina kutnjaka; (2) upis u jednu od deset odabranih škola; (3) dob između šest i osam godina; (4) bez fiksnih ortodontskih naprava; (5) pristanak djeteta na stomatološke preglede. 


\section{Intervention}

Once participants were selected, we performed an initial randomization to determine which sealant would be used for each child. We then performed the second randomization to determine on which side of the mouth the sealant would be placed. Sealants (experimental group) were applied in the first upper permanent molar and first lower permanent molar on the same side of the mouth. In this way, the control group was assigned to the opposite arch.

\section{Data collection and statistical analysis}

We used the International Caries Detection and Assessment System II (ICDAS II) to measure caries experience at baseline and follow-up (six months after intervention); $\mathrm{dmft}$ index was used at baseline as well. Both the clinical examiner and recorder were calibrated and standardized (kappa >0.80) on this scale. As recommended by other authors (17), we assessed pit and fissure sealants during follow-up as $0=$ intact or complete, $1=$ partial retention, or $2=$ missing.

After describing general characteristics of the sample, we performed a bivariate analysis to ascertain whether differences had arisen between the two groups of participants in terms of loss to follow-up, and for each of the independent variables. We performed a chi square test to establish the association between dependent and exposure variables. Finally, we estimated the relative risk (RR) and the incidence of caries in the FPMs with and without a sealant. Given the split-mouth design of our study, the tooth was analyzed so that a comparison could be made with the contralateral tooth.

\section{Variables}

Our dependent variable was the incidence of caries, measured dichotomously $(0=$ sound, $1=$ visible changes in enamel or caries $>1$ in ICDAS II). Our main independent variable was the presence of sealant $(0=$ without sealant, $1=$ with sealant). In addition, we analyzed data by sex $(0=$ male, $1=\mathrm{fe}-$ male), age (6-8 years), baseline $\mathrm{dmft}$, type of sealant $(0=\mathrm{Clin}-$ pro, $1=$ BeautiSealant) and sealant status six months after placement ( 0 =intact, $1=$ partial retention, $2=$ missing).

\section{Blinding}

The simple blind design was used; i.e., participants were unaware of group assignment, but researchers were aware of it. Similarly, both clinicians and patients knew that the treatment would be provided, but patients did not know which type of sealant would be applied. The dentists placing the sealants were different from those scoring the caries experience, the presence of sealants, or collecting data for the trial.

\section{Results}

Table 1 shows the general sample and demographic characteristics. Of 140 participants, mean of age was $6.92 \pm 0.74$ years; $52.1 \%$ were boys. Mean baseline $\mathrm{dmft}$ index was $4.10 \pm 3.16$. Although the study had a $12.9 \%$ loss to followup, no statistically significant differences $(p>0.05)$ were observed for sex, age, baseline dmft nor type of sealants between

\section{Postupak}

Nakon odabira sudionika obavili smo početnu randomizaciju kako bismo utvrdili koja će se pečatna smola upotrebljavati za svako dijete. Zatim smo učinili drugu randomizaciju i utvrdili na koju će se stranu usta postaviti pečatna smola. Pečatne smole (eksperimentalna skupina) primijenjene su u gornjem prvom trajnom kutnjaku i donjem prvom trajnom kutnjaku na istoj strani usta.

\section{Prikupljanje podataka i statistička analiza}

Koristili smo se međunarodnim sustavom za otkrivanje i procjenu karijesa II (ICDAS II) za mjerenje prevalencije karijesa na početku praćenja i tijekom praćenja (šest mjeseci nakon intervencije). DMFT indeks također je upotrijebljen na početku. Klinički ispitivači su kalibrirani i standardizirani (kappa > 0,80) na ovoj ljestvici. Prema preporuci drugih autora (17), smole za pečaćenje fisura i jamica tijekom praćenja ocjenjivali smo sljedećim ocjenama: 0 = intaktne ili potpune, 1 = djelomična retencija ili $2=$ nedostaje.

Nakon opisa općih karakteristika uzorka proveli smo bivarijantnu analizu kako bismo ustanovili jesu li se pojavile razlike između dviju skupina sudionika u smislu gubitka praćenja i za svaku od neovisnih varijabli. Proveli smo hi-kvadrat test kako bismo utvrdili povezanost između ovisnih i varijabli izloženosti. Na kraju smo procijenili relativni rizik (RR) i prevalenciju karijesa na prvim trajnim kutnjacima s brtvilom i bez njega. S obzirom na dizajn usta s podijeljenim ustima, analiza je provedena zub po zub da bi se mogla napraviti usporedba s kontralateralnim zubom.

\section{Varijable}

Naša ovisna varijabla bila je incidencija karijesa mjerena dihotomno ( 0 = zdravo, 1 = vidljive promjene cakline ili karijes > 1 prema ICDAS-u II). Naša glavna neovisna varijabla bila je prisutnost pečata $(0=$ bez pečata, $1=s$ pečatom $)$. $U_{z}$ to, analizirali smo podatke prema spolu $(0=$ muški, $1=$ ženski), dobi (6 - 8 godina), osnovnom DMFT-u, vrsti pečatne smole $(0$ = Clinpro, 1 = BeautiSealant $)$ i statusu pečata šest mjeseci nakon postavljanja $(0=$ intaktno, $1=$ djelomična retencija, 2 = nedostaje).

\section{Zasljepljivanje}

Primijenjen je jednostavan dizajn zasljepljivanja, tj. sudionici nisu bili svjesni rasporedivanja po skupinama, ali istraživači jesu. I kliničari i pacijenti znali su da će se obaviti liječenje, ali pacijenti nisu znali koja će se vrsta smole za pečaćenje primijeniti. Stomatolozi koji su pečatili zube nisu bili oni isti koji su bilježili incidenciju karijesa, prisutnost pečatne smole i prikupljali podatke za istraživanje.

\section{Rezultati}

Tablica 1. općenito opisuje uzorak i demografske karakteristike. Među 140 sudionika srednja dob iznosila je 6,92 \pm 0,74 godine $-52,1 \%$ bili su dječaci. Prosječni DMFT indeks bio je na početku 4,10 $\pm 3,16$. Iako je tijekom istraživanja zabilježen odlazak ispitanika od 12,9\%, nisu uočene statistički značajne razlike $(\mathrm{p}>0,05)$ za spol, dob, početni DMFT, ni za 
Table 1 General sample characteristics at baseline and analysis of participants who remained and did not remain (loss to follow-up) in the study

Tablica 1. Opće karakteristike uzorka na početku istraživanja i analiza sudionika koji su ostali i koji nisu ostali u istraživanju (gubitak praćenja)

\begin{tabular}{|c|c|c|c|c|c|}
\hline Variable $\bullet$ Varijabla & \multicolumn{2}{|c|}{$\begin{array}{c}\text { Baseline measurement } \\
\text { Početno mjerenje } \\
\text { n (\%) }\end{array}$} & $\begin{array}{l}\text { Lost to follow up } \\
\text { Gubitak praćenja } \\
18(12.9)\end{array}$ & $\begin{array}{l}\text { Remained in study } \\
\text { Ostali u istraživanju } \\
\quad 122(87.1)\end{array}$ & $P$ value $\bullet P$ vrijednost \\
\hline $\begin{array}{l}\text { Sex } \bullet \text { Spol } \\
\text { Male } \bullet \text { Muški } \\
\text { Female } \bullet \text { Ženski } \\
\end{array}$ & \multicolumn{2}{|c|}{$\begin{array}{l}73(52.1) \\
67(47.9)\end{array}$} & $\begin{array}{l}11(15.1) \\
7(10.4)\end{array}$ & $\begin{array}{l}62(84.9) \\
60(89.6)\end{array}$ & $\mathrm{p}=0.415^{*}$ \\
\hline $\begin{array}{l}\text { Type of sealant } \bullet \text { Vrsta smole za pečaćenje } \\
\text { Beautisealant } \\
\text { Clintpro }\end{array}$ & \multicolumn{2}{|c|}{$\begin{array}{l}70(50.0) \\
70(50.0)\end{array}$} & $\begin{array}{c}11(8.4) \\
10(14.3)\end{array}$ & $\begin{array}{l}62(88.6) \\
60(85.7)\end{array}$ & $\mathrm{p}=0.614^{*}$ \\
\hline & $\begin{array}{l}\text { Mean } \pm \text { SD } \\
\text { Srednja vrijednost } \\
\pm \text { SD }\end{array}$ & $\begin{array}{l}\text { Range } \\
\text { Raspon }\end{array}$ & $\begin{array}{c}\text { Mean } \pm S D \cdot \text { Srednja } \\
\text { vrijednost } \pm S D\end{array}$ & $\begin{array}{c}\text { Mean } \pm S D \bullet \text { Srednja } \\
\text { vrijednost } \pm S D\end{array}$ & \\
\hline Age $\bullet$ Dob & $6.92 \pm 0.74$ & $6-8$ & $6.66 \pm 0.68$ & $6.96 \pm .74$ & $\mathrm{p}=0.1117^{* *}$ \\
\hline Baseline dmft • Početni DMFT & $4.10 \pm 3.16$ & $0-13$ & $4.33 \pm 3.49$ & $4.06 \pm 3.12$ & $\mathrm{p}=0.8095^{* *}$ \\
\hline
\end{tabular}

\begin{tabular}{|c|c|c|c|}
\hline FPM16・PTK16 & Sound $\bullet$ Zdravo & Decayed $\bullet$ Karijes & $P$ value $\bullet P$ vrijednost \\
\hline $\begin{array}{c}\text { Presence of sealant } \bullet \text { Prisutnost pečata } \\
\text { With sealant } \bullet S \text { pečatom } \\
\text { Without sealant } \bullet \text { Bez pečata } \\
\end{array}$ & $\begin{array}{l}43(74.1) \\
32(50.0)\end{array}$ & $\begin{array}{l}15(25.9) \\
32(50.0)\end{array}$ & $\mathrm{p}=0.006^{*}$ \\
\hline $\begin{array}{l}\text { Sealant status } \bullet \text { Status pečata } \\
\text { Intact } \bullet \text { Intaktan } \\
\text { Partial } \bullet \text { Djelomično očuvan } \\
\text { Missing } \bullet \text { Izgubljen } \\
\end{array}$ & $\begin{array}{l}36(81.8) \\
28(60.9) \\
11(34.4)\end{array}$ & $\begin{array}{l}8(18.2) \\
18(39.1) \\
21(65.6)\end{array}$ & $\mathrm{p}<0.0001^{*}$ \\
\hline $\begin{array}{l}\text { Type of sealant } \bullet \text { Vrsta smole za pečaćenje } \\
\text { Clinpro } \\
\text { BeautiSealant }\end{array}$ & $\begin{array}{l}45(75.0) \\
30(48.4)\end{array}$ & $\begin{array}{l}15(25.0) \\
32(51.6)\end{array}$ & $\mathrm{p}=0.003^{*}$ \\
\hline $\begin{array}{l}\text { Sex } \bullet \text { Spol } \\
\text { Male } \bullet \text { Muški } \\
\text { Female } \bullet \text { Ženski } \\
\end{array}$ & $\begin{array}{l}38(61.3) \\
37(61.7) \\
\end{array}$ & $\begin{array}{l}24(38.6) \\
23(38.3)\end{array}$ & $\mathrm{p}=0.966^{*}$ \\
\hline Age $\bullet$ Dob & $6.89 \pm 0.70$ & $7.08 \pm 0.80$ & $\mathrm{p}=0.1757^{* *}$ \\
\hline Baseline $\mathrm{dmft} \cdot$ Početni DMFT & $3.74 \pm 3.08$ & $4.57 \pm 3.15$ & $\mathrm{p}=0.1336^{* *}$ \\
\hline \multicolumn{4}{|l|}{ FPM26・PTK26 } \\
\hline $\begin{array}{c}\text { Presence of sealant } \bullet \text { Prisutnost pečata } \\
\text { With sealant } \bullet S \text { pečatom } \\
\text { Without sealant } \bullet \text { Bez pečata } \\
\end{array}$ & $\begin{array}{l}51(79.7) \\
17(29.3) \\
\end{array}$ & $\begin{array}{l}13(20.3) \\
41(70.7) \\
\end{array}$ & $\mathrm{p}<0.0001^{*}$ \\
\hline $\begin{array}{l}\text { Sealant status } \bullet \text { Status pečata } \\
\text { Intact } \bullet \text { Intaktan } \\
\text { Partial } \bullet \text { Djelomično očuvan } \\
\text { Missing } \bullet \text { Izgubljen } \\
\end{array}$ & $\begin{array}{l}28(63.6) \\
27(58.7) \\
13(40.6)\end{array}$ & $\begin{array}{l}16(36.4) \\
19(41.3) \\
19(59.4) \\
\end{array}$ & $\mathrm{p}=0.120^{*}$ \\
\hline $\begin{array}{l}\text { Type of sealant } \bullet \text { Vrsta smole za pečaćenje } \\
\text { Clinpro } \\
\text { BeautiSealant } \\
\end{array}$ & $\begin{array}{l}36(60.0) \\
32(51.6) \\
\end{array}$ & $\begin{array}{l}24(40.0) \\
30(48.4) \\
\end{array}$ & $\mathrm{p}=0.351^{*}$ \\
\hline $\begin{array}{l}\text { Sex } \bullet \text { Spol } \\
\quad \text { Male } \bullet \text { Muški } \\
\text { Female } \bullet \text { Ženski }\end{array}$ & $\begin{array}{l}32(51.6) \\
36(60.0)\end{array}$ & $\begin{array}{l}30(48.4) \\
24(40.0)\end{array}$ & $\mathrm{p}=0.351^{*}$ \\
\hline Age $\bullet$ Dob & $6.97 \pm 0.71$ & $6.96 \pm 0.80$ & $\mathrm{p}=0.9383^{* *}$ \\
\hline Baseline $\mathrm{dmft} \bullet$ Početni DMFT & $3.52 \pm 2.76$ & $4.74 \pm 3.44$ & $\mathrm{p}=0.0489^{* *}$ \\
\hline
\end{tabular}

${ }^{*} \mathrm{Chi}^{2} ;{ }^{* *}$ Man-Withney; FPM $\bullet$ PTK $=$ first permanent molar $\bullet$ prvi trajni kutnjak

children who left the study and those who completed the follow-up.

There were no statistically significant differences $(\mathrm{p}>0.05)$ in caries incidence in FPMs by age or sex. A lower caries incidence was observed in FPMs with pit and fissure sealants $(p<0.01)$ (Tables 2 and 3), regardless of the type used. vrstu pečatne smole između djece koja su napustila istraživanje i one koja su završila praćenje.

Nije bilo statistički značajnih razlika $(p>0,05)$ u incidenciji karijesa na prvim trajnim kutnjacima ni prema dobi, ni prema spolu. Manja incidencija karijesa zabilježena je na prvim trajnim kutnjacima sa zapečaćenim fisurama $(\mathrm{p}<$ 
Table 3 Caries incidence in FPM36 and FPM46 vs independent variables

Tablica 3. Incidencija karijesa na prvim trajnim kutnjacima 36 i 46 u usporedbi s neovisnim varijablama

\section{FPM36 • PTK36}

Presence of sealant $\bullet$ Prisutnost pečata

With sealant $\bullet S$ pečatom

Without sealant $\bullet$ Bez pečata

Sealant status $\bullet$ Status pečata

Intact $\bullet$ Intaktan

Partial • Djelomično očuvan

Missing • Izgubljen

Sound $\bullet$ Zdravo

Decayed • Karijes

$P$ value $\bullet P$ vrijednost

Type of sealant $\bullet$ Vrsta smole za pečaćenje

Clinpro

BeautiSealant

Sex $\bullet$ Spol

Male $\bullet$ Muški

Female • Ženski

Age $\bullet$ Dob

Baseline dmft $\bullet$ Početni DMFT

FPM46・PTK46

Presence of sealant $\bullet$ Prisutnost pečata

With sealant $\bullet S$ pečatom

Without sealant $\bullet$ Bez pečata

Sealant status $\bullet$ Status pečata

Intact $\bullet$ Intaktan

Partial $\bullet$ Djelomično očuvan

Missing • Izgubljen

Type of sealant $\bullet$ Vrsta smole za pečaćenje

Clinpro

BeautiSealant

Sex $\bullet$ Spol

Male $\bullet$ Muški

Female $\bullet$ Ženski

Age $\bullet$ Dob

Baseline $\mathrm{dmft} \bullet$ Početni DMFT

\begin{tabular}{|c|c|c|}
\hline Sound $\bullet Z$ Zdravo & Decayed • Karijes & $P$ value $\bullet P$ vrijednost \\
\hline $\begin{array}{l}50(78.1) \\
21(36.2) \\
\end{array}$ & $\begin{array}{l}14(21.9) \\
37(63.8) \\
\end{array}$ & $\mathrm{p}<0.0001^{*}$ \\
\hline $\begin{array}{l}27(61.4) \\
30(65.2) \\
14(43.7)\end{array}$ & $\begin{array}{l}17(38.6) \\
16(34.8) \\
18(56.3)\end{array}$ & $\mathrm{p}=0.145^{*}$ \\
\hline $\begin{array}{l}41(68.3) \\
30(48.4) \\
\end{array}$ & $\begin{array}{l}19(31.7) \\
32(51.6) \\
\end{array}$ & $\mathrm{p}=0.026^{*}$ \\
\hline $\begin{array}{l}37(59.7) \\
34(56.7) \\
\end{array}$ & $\begin{array}{l}25(40.3) \\
26(43.3) \\
\end{array}$ & $\mathrm{p}=0.736^{*}$ \\
\hline $6.92 \pm .72$ & $7.01 \pm .78$ & $\mathrm{p}=0.5221^{* *}$ \\
\hline $3.33 \pm 2.49$ & $5.07 \pm 3.62$ & $\mathrm{p}=0.0094^{* *}$ \\
\hline $\begin{array}{l}43(74.1) \\
27(42.2)\end{array}$ & $\begin{array}{l}15(25.9) \\
37(57.8) \\
\end{array}$ & $\mathrm{p}<0.0001^{*}$ \\
\hline $\begin{array}{l}28(63.6) \\
27(58.7) \\
15(46.9)\end{array}$ & $\begin{array}{l}16(36.4) \\
19(41.3) \\
17(53.1)\end{array}$ & $\mathrm{p}=0.336^{*}$ \\
\hline $\begin{array}{l}41(68.3) \\
29(46.8) \\
\end{array}$ & $\begin{array}{l}19(31.7) \\
33(53.2) \\
\end{array}$ & $\mathrm{p}=0.016^{*}$ \\
\hline $\begin{array}{l}38(61.3) \\
32(53.3) \\
\end{array}$ & $\begin{array}{l}24(38.7) \\
28(46.7) \\
\end{array}$ & $\mathrm{p}=0.314^{*}$ \\
\hline $6.87 \pm .74$ & $7.09 \pm .74$ & $\mathrm{p}=0.1015^{* *}$ \\
\hline $3.71 \pm 3.11$ & $4.53 \pm 3.1$ & $\mathrm{p}=0.1147^{* *}$ \\
\hline
\end{tabular}

${ }^{*} \mathrm{Chi}^{2}$; ${ }^{* *}$ Man-Withney; FPM $\bullet$ PTK $=$ first permanent molar $\bullet$ prvi trajni kutnjak

\begin{tabular}{|c|c|c|c|c|}
\hline FPM16・PTK16 & Sound $\bullet$ Zdravo & $\begin{array}{l}\text { ICDAS } 1 \text { and } 2 \\
\text { ICDAS } 1 \text { i } 2\end{array}$ & ICDAS 3 & $P$ value $\bullet P$ vrijednost \\
\hline $\begin{array}{l}\text { Presence of sealant } \bullet \text { Prisutnost pečata } \\
\text { With sealant } \bullet S \text { pečatom } \\
\text { Without sealant } \bullet \text { Bez pečata }\end{array}$ & $\begin{array}{l}43(74.1) \\
32(50.0)\end{array}$ & $\begin{array}{c}9(15.5) \\
21(32.8)\end{array}$ & $\begin{array}{c}6(10.4) \\
11(17.2)\end{array}$ & $\mathrm{p}=0.022^{*}$ \\
\hline \multicolumn{5}{|l|}{ FPM26・PTK26 } \\
\hline $\begin{array}{l}\text { Presence of sealant } \bullet \text { Prisutnost pečata } \\
\text { With sealant } \bullet S \text { pečatom } \\
\text { Without sealant } \bullet \text { Bez pečata }\end{array}$ & $\begin{array}{l}51(79.7) \\
17(29.3)\end{array}$ & $\begin{array}{l}10(15.6) \\
32(55.2)\end{array}$ & $\begin{array}{c}3(4.7) \\
9(15.5)\end{array}$ & $\mathrm{p}<0.0001^{*}$ \\
\hline \multicolumn{5}{|l|}{ FPM36・PTK36 } \\
\hline $\begin{array}{l}\text { Presence of sealant } \bullet \text { Prisutnost pečata } \\
\text { With sealant } \bullet S \text { pečatom } \\
\text { Without sealant } \bullet \text { Bez pečata }\end{array}$ & $\begin{array}{l}50(78.1) \\
21(36.2)\end{array}$ & $\begin{array}{l}10(15.6) \\
18(31.0)\end{array}$ & $\begin{array}{c}4(6.3) \\
19(32.8)\end{array}$ & $\mathrm{p}<0.0001^{*}$ \\
\hline \multicolumn{5}{|l|}{ FPM46・PTK 46} \\
\hline $\begin{array}{l}\text { Presence of sealant } \bullet \text { Prisutnost pečata } \\
\text { With sealant } \bullet S \text { pečatom } \\
\text { Without sealant } \bullet \text { Bez pečata }\end{array}$ & $\begin{array}{l}43(74.1) \\
27(42.2)\end{array}$ & $\begin{array}{c}9(15.5) \\
16(25.0)\end{array}$ & $\begin{array}{c}6(10.4) \\
21(32.8)\end{array}$ & $\mathrm{p}=0.001^{*}$ \\
\hline
\end{tabular}

${ }^{*}$ Chi square $\bullet$ Hi-kvadrat

When sealants remained intact there was a lower caries incidence compared to sealants partially or completely missing - but differences were only significant for FPM 16. The caries incidence was higher for BeautiSealant sealed teeth than
0,01) (tablice 2. i 3.), bez obzira na upotrijebljenu pečatnu smolu.

Kada je pečat ostao intaktan, bila je manja incidencija karijesa u usporedbi s djelomično ili potpuno nestalim pečati- 
Table 5 Caries incidence and relative risk in teeth with sealant

Tablica 5. Incidencija karijesa i relativni rizik na zapečaćenim zubima

\begin{tabular}{|c|c|c|c|c|c|c|}
\hline & $\begin{array}{l}\text { IR - exposed • } \\
\text { IR - izložen }\end{array}$ & $\begin{array}{l}\text { IR - non-exposed } \\
\text { IR - neizložen }\end{array}$ & CI & RR & CI $95 \%$ & $\begin{array}{c}\text { P value } \bullet \\
\text { P vrijednost }\end{array}$ \\
\hline FPM16• PTK16 & 25.0 & 50.0 & 38.5 & 0.52 & $0.52-0.85$ & $\mathrm{p}=0.0062$ \\
\hline FPM26・PTK26 & 20.0 & 70.0 & 42.2 & 0.29 & $0.17-0.48$ & $\mathrm{p}<0.0001$ \\
\hline FPM36・PTK36 & 21.8 & 63.7 & 41.8 & 0.34 & $0.21-0.57$ & $\mathrm{p}<0.0001$ \\
\hline FPM46• PTK46 & 25.8 & 57.8 & 42.6 & 0.45 & $0.27-0.72$ & $\mathrm{p}=0.0004$ \\
\hline
\end{tabular}

$\mathrm{PTK}=$ prvi trajni kutnjak; $\mathrm{IR}=$ Incidence rate $\bullet$ stopa incidencije; $\mathrm{CI}=$ Cumulative incidence $\bullet$ kumulativna incidencija; $\mathrm{RR}=\mathrm{Relative} \mathrm{risk} \bullet$ relativni rizik

Clinpro's, but it was only statistically significant in FPMs 16, 36 and $46(\mathrm{p}<0.05)$. The caries incidence was higher in those cases with a higher baseline $\mathrm{dmft}$ but it only reached statistical significance in FPMs 26 and 36.

Table 4 shows the incidence distribution by ICDAS II type of lesion. They were primarily ICDAS 1 and 2; 63.8\% of 47 FPM 16, 77.7\% of 54 FPM 26, 54.9\% of 51 FPM 36 and $48.0 \%$ of FPM 46.

The caries incidence in sealed and non-sealed FPMs is presented in Table 5. In terms of relative risk (RR), FPMs with pit and fissure sealants had lower caries risk of than those without sealants.

\section{Discussion}

Our study demonstrated that pit and fissure sealants are effective for a six-month follow-up period when applied to first permanent molars (FPMs). This is consistent with previous studies in various countries $(18,19)$, although the followup times differ. While widely acknowledged for effectiveness, it is a pity that in Mexico pit and fissure sealants are not employed as often as it could be expected. According to a report from the Ministry of Health, less than $2.5 \%$ of the population surveyed had received sealants (11). Given demonstrated effectiveness, health programs should be emphasizing sealant utilization in public and private dental services, as it has often been recommended in other countries $(18,19)$.

Caries lesions are predictive of new ones (20); a previous history of caries experience suggests an increased risk (21). Pit and fissure sealants are especially indicated for children at risk of developing caries. When we analyzed the relationship between the initial appearance of caries in primary dentition and the incidence of caries in sealed teeth, we found higher incidence of caries in patients with greater $\mathrm{dmft}$ at baseline. Despite our limited follow-up time, overall results strongly support the use of sealants $(15,17-19)$.

The BeautiSelant@Shofu sealant utilizes glass ionomer together with a polymer in its composition, which offers the advantage of releasing and recharging fluoride. Recent studies have compared this type of technology to conventional sealants with favorable results $(22,23)$. Cinpro@ $3 \mathrm{M}$ is a resinbased sealant that releases fluoride. It has been suggested that this type of sealant provides greater benefits as it tends to remain longer in the mouth (24): resin-based sealants offer extended retention as a result of the adhesive system used (25). Our study results showed a smaller number of missing seal- ma, no razlika je bila značajna samo za zub 16. Incidencija karijesa bila je veća na zubima zapečaćenima smolom BeautiSealant, nego smolom Clinproove, ali je razlika bila statistički značajna samo za zube 16, 36 i 46 ( $p<0,05)$. Incidencija karijesa bila je veća u slučajevima s višim početnim DMFT-om, ali je statistički značajna bila samo za zube 26 i 36.

U tablici 4. nalazi se distribucija incidencije prema tipu lezije ICDAS II. Uglavnom su to bili ICDAS 1 i 2; 63,8 \% od 47 na zubu 16, 77,7 \% od 54 na zubu 26, 54,9\% od 51 na zubu 36 i 48,0\% na zubu 46.

Incidencija karijesa na zapečaćenim i nezapečaćenim prvim trajnim kutnjacima prikazana je u tablici 5 . Kad je riječ o relativnom riziku (RR), zapečaćeni prvi trajni kutnjaci imali su manji rizik od nastanka karijesa od onih bez pečata.

\section{Rasprava}

Naše istraživanje pokazalo je da su smole za pečaćenje fisura i jamica učinkovite tijekom šestomjesečnog razdoblja praćenja kada se primjenjuju na prvim trajnim kutnjacima (FPM). To je u skladu s rezultatima dosadašnjih istraživanja u raznim zemljama $(18,19)$, iako se vrijeme praćenja razlikuje. Iako je dokazana učinkovitost, u Meksiku se, nažalost, smole za pečaćenje fisura i jamica ne primjenjuju toliko često koliko bi se moglo očekivati. Prema izvješću Ministarstva zdravstva, manje od 2,5\% ispitane populacije ima zapečaćene fisure (11). S obzirom na dokazanu učinkovitost, zdravstveni programi trebali bi isticati korist od pečaćenja zuba u javnim i privatnim stomatološkim ordinacijama, kao što se često preporučuje u drugim zemljama $(18,19)$.

Postojeće karijesne lezije prediktivni su čimbenik za nastanak novih (20); povijest karijesa upućuje na povećan rizik (21). Smole za pečaćenje fisura i jamica posebno su indicirane za djecu kojoj prijeti karijes. Kada smo analizirali vezu između inicijalne pojave karijesa u mliječnoj denticiji i incidencije karijesa na zapečaćenim zubima, ustanovili smo veću učestalost kod pacijenata s većim DMFT indeksom na početku. Unatoč našem ograničenom vremenu praćenja, ukupni rezultati snažno podupiru primjenu smola za pečaćenje $(15,17-19)$.

Smola za pečaćenje BeautiSelant ${ }^{\oplus}$ Shofu sadržava stakleni ionomer zajedno s polimerom, što ima prednost oslobađanja i punjenja fluorida. Nedavno provedena istraživanja uspoređivala su tu vrstu tehnologije s konvencionalnim smolama za pečaćenje s povoljnim rezultatima $(22,23)$. Cinpro ${ }^{\odot} 3 \mathrm{M}$ bazira se na smoli koja oslobađa fluor. Sugerira se da je ta vrsta smole za pečaćenje korisnija jer obično ostaje dulje u ustima (24): takve smole za pečaćenje imaju produženu retenciju 
ants and a lower level of caries in FPMs when Clinpro@3M sealants had been used. The effectiveness of pit and fissure sealants is closely related to their permanence on the tooth surface (26-28). However, we found no differences in terms of the incidence of caries in relation to retention. Our study differed from others because our follow-up measurement was limited to six months; the effect of total or partial loss of sealants may have been more evident over a longer period.

While selection criteria included four first permanent molars in good shape, such condition may have not ensured good overall dental health status; primary teeth could have had caries. In Table 1, we had dmft 4.10 \pm 3.16 , which is considerable. Through resorting to ICDAS II, we were able to identify enamel changes, from minimal color changes indicating demineralization (ICDAS 1 and 2) to carious lesions encompassing small cavities as well as extensive enamel breakdown (ICDAS $>2$ ). Most lesions scored were ICDAS 1 and 2 , a few ICDAS 3 , and none above ICDAS 3.

Pit and fissure sealants can be an effective tool to prevent dental caries and thus help improve oral health of the child population. Our research findings support sealant utilization in one of the prime age groups who can benefit from such a caries prevention approach $(7-10,14-19,28-30)$.

\section{Conclusions}

Pit and fissure sealants offer an effective preventive treatment for reducing the incidence of caries over a six-month follow-up period in six to eight years old schoolchildren, regardless of either one of two types of sealants used. In our study, the sealant that proved to be most effective in terms of prevention and retention was Clinpro@3M.

\section{Acknowledgements}

This study was supported by a scholarship awarded by the National Council of Science and Technology of Mexico (CONACYT) to MAFB.

\section{Competing financial interests}

The authors state that there are no actual or perceived conflicts of interest. The authors received no product support or any kind of financial support from the manufacturers of the sealants.

Author's Contribution: M.A.F.-B., T.de J.S.-M., C.E.M.-S., and G.M., conceptualized and designed the study. R.J.S.-V., and MdeL.M.C., contributed to data analysis and interpretation of data. All authors contributed to a critical review of the manuscript, writing the first draft and approving the final version. All authors have read and agreed to the published version of the manuscript. kao rezultat upotrijebljenog adhezivnog sustava (25). Rezultati našeg istraživanja pokazali su manje izgubljenih pečata i nižu razinu karijesa na prvim trajnim kutnjacima kada je korištena smola Clinpro $^{\oplus} 3 \mathrm{M}$. Učinkovitost smola za pečaćenje fisura i jamica usko je povezana s njihovom postojanošću na površini zuba $(26-28)$. No nismo pronašli razlike u vezi s incidencijom karijesa u odnosu prema retenciji. Naše istraživanje razlikovalo se od ostalih zato što je praćenje bilo ograničeno na šest mjeseci; učinak potpunog ili djelomičnog gubitka pečata mogao je biti očitiji tijekom duljeg razdoblja praćenja.

Iako je kriterij za uključivanje bio četiri zdrava prva trajna kutnjaka, takvo stanje možda zapravo nije jamčilo dobro ukupno zdravlje zuba - mliječni zubi mogli su imati karijes. U tablici 1. DMFT je iznosio 4,10 $\pm 3,16$, što je prilično visoko. $S$ pomoću klasifikacije ICDAS II uspjeli smo identificirati promjene cakline - od minimalnih promjena boje koje upućuju na demineralizaciju (ICDAS 1 i 2) do karijesnih lezija s malim kavitetima te opsežno urušavanje cakline (ICDAS > 2). Većina lezija bile su ICDAS 1 i 2, nekoliko ICDAS 3 i ni jedna nije bila iznad ICDAS-a 3.

Smole za pečaćenje fisura i jamica mogu biti učinkovito sredstvo za prevenciju karijesa i tako poboljšati oralno zdravlje dječje populacije. Nalazi u našem istraživanju idu u prilog pečaćenju fisura u jednoj od najvažnijih dobnih skupina koje imaju korist od takvog pristupa prevenciji karijesa (7 - 10, $14-19,28-30)$.

\section{Zaključci}

Smole za pečaćenje fisura i jamica učinkovit su preventivni tretman za smanjenje incidencije karijesa tijekom šestomjesečnog razdoblja praćenja kod šestogodišnjaka, neovisno o kojoj je od dviju vrsta pečatne smole riječ. $U$ našem istraživanju smola koja se pokazala učinkovitijom, kad je riječ o prevenciji i retenciji, bio je $\mathrm{Clinpro}^{\oplus} 3 \mathrm{M}$.

\section{Zahvala}

Ovo je istraživanje financirano stipendijom koju je $\mathrm{Na}$ cionalno vijeće za znanost i tehnologiju u Meksiku (CONACYT) dodijelilo MAFB-u.

\section{Sukob interesa}

Autori navode da nisu bili u sukobu interesa. Nisu dobili ni proizvode, ni bilo kakvu financijsku potporu od proizvođača smola za pečaćenje.

Doprinos autora: M. A. F.-B., T. de J. S.-M., C. E. M.- S., G. M. - koncept i dizajn istraživanja. R. J. S.-V., Mde L. M.-C. - doprinos analizi i interpretaciji podataka. Napisavši prvi nacrt i odobrivši konačnu verziju, svi su autori dali svoj doprinos kritičkom pregledu rukopisa. Tako đer su ga svi pročitali i složili se s verzijom rukopisa za objavljivanje. 


\section{Sažetak}

Svrha rada: Procjena učinkovitosti smola za pečaćenje jamica i fisura (PFS) u smanjenju učestalo sti zubnog karijesa kod školaraca. Materijali i metode: Primijenjen je randomizirani eksperimentalni dizajn s podijeljenim ustima u uzorku od 140 ispitanika raspoređenih u dvije skupine. Korištene su smole za pečaćenje Clinpro ${ }^{5} 3 \mathrm{M}$ i BeautiSealant ${ }^{5}$ Shofu koje su aplicirane na prve trajne kutnjake (FPM). Sve smole uspoređene su s kutnjacima na kontrolnoj strani kako bi se ustanovila učinkovitost tijekom 6 mjeseci. U istraživanju je postotak ispitanika koji su odustali od praćenja iznosio 12,9 \%. Nisu uočene statistički značajne razlike $(p>0,05)$ za spol, dob, početni DMFT ili vrstu smole. Za statističku analizu upotrijebljeni su neparametrijski testovi. Rezultati : Prosječni DMFT indeks bio je na početku 4,10 $\pm 3,16$. Manja incidencija karijesa zabilježena je na zapečaćenim prvim trajnim kutnjacima $(p<0,01)$, bez obzira na vrstu smole. Kada su smole ostale intaktne, incidencija karijesa bila je manja u usporedbi s djelomično ili potpuno nestalim pečatima, no razlika je bila statistički značajna samo za prve trajne kutnjake 16. Incidencija karijesa bila je veća kod zuba zapečaćenih smolom BeautiSealant u usporedbi sa smolom Clinpro, ali je statistički značajna bila samo kod prvih trajnih kutnjaka 16, 36 i 46 ( $p<0,05)$. Incidencija karijesa bila je veća u slučajevima s višim početnim DMFT-om, ali je statistički značajna samo za prve trajne kutnjake 26 i 36. Relativni rizik od karijesa bio je niž kod zapečaćenih zuba $(\mathrm{p}<0,01)$. Zaključak: Smole za pečaćenje fisura i jamica učinkovite su u prevenciji karijesa tijekom šestomjesečnog razdoblja praćenja školske djece od 6 do 8 godina, bez obzira na vrstu smole. Smola s većom učinkovitošću, kad je riječ o prevenciji i retenciji, bio je Clinproš $3 M$.
Zaprimljen: 6. srpnja 2020.

Prihvaćen: 24 . veljače 2021

Adresa za dopisivanje

LMr. sc. Carlo Eduardo Medina-Solís

Avenida Álamo \# 204

Fraccionamiento Paseo de los Solares

CP. 42113. Pachuca de Soto, Hidalgo,

Mexico

cemedinas@yahoo.com

MeSH pojmovi: materijali za pečaćenje fisura; zubni karijes; dijete Ključne riječi: smole za pečaćenje fisura i jamica, oralno zdravlje, karijes

\section{References}

1. Kassebaum NJ, Bernabé E, Dahiya M, Bhandari B, Murray CJL, Marcenes W. Global burden of untreated caries: a systematic review and metaregression. J Dent Res. 2015 May;94(5):650-8.

2. Martins-Paiva S, Álvarez-Vidigal E, Abanto J, Cabrera-Matta A, López-Robles RA, Masoli C, et al. Epidemiology of dental caries in Latin America. Revista de Odontopediatría Latinoamericana 2014; 4(2). Available from: https://www.revistaodontopediatria. org/ediciones/2014/2/art-4/.

3. MeSH Browser [database on the Internet]. Ministry of Health. National Caries Survey 2001. Centro Nacional de Vigilancia Epidemiológica y Control de Enfermedades. México DF; 2006. Available from: https://www.paho.org/hq/dmdocuments/2009/OralHealthPart3.pdf.

4. Medina-Solís CE, Ávila-Burgos L, Márquez-Corona ML, MedinaSolís JJ, Lucas-Rincón SE, Borges-Yañez SA, et al. Out-of-pocket expenditures on dental care for schoolchildren aged 6 to 12 years: A cross-sectional estimate in a less-developed country setting. Int J Environ Res Public Health. 2019 Jun 5;16(11):1997.

5. Medina-Solís CE, Ávila-Burgos L, Borges-Yañez SA, IrigoyenCamacho ME, Sánchez-Pérez L, Zepeda-Zepeda MA, et al. Ecological study on needs and cost of treatment for dental caries in schoolchildren aged 6,12 and 15 years: Data from a national survey in Mexico. Medicine (Baltimore). 2020 Feb;99(7):e19092.

6. Pontigo-Loyola AP, Márquez-Corona ML, Minaya-Sánchez M, Lucas-Rincón SE, Casanova-Rosado JF, Robles-Minaya JL, et al. Correlation between the caries status of the first permanent molars and the overall DMFT Index: a cross-sectional study. Medicine (Baltimore). 2020 Jan;99(5):e19061.

7. Oz FD, Ergin E, Cakir FY, Gurgan S. Clinical Evaluation of a SelfAdhering Flowable Resin Composite in Minimally Invasive Class I Cavities: 5-year Results of a Double Blind Randomized, Controlled Clinical Trial. Acta Stomatol Croat. 2020 Mar;54(1):10-21.

8. Colombo S, Paglia L. Dental Sealants. Part 1: Prevention First. Eur J Paediatr Dent. 2018 Mar;19(1):80-82.

9. Hatibović-Kofman S, El-Kassem M, Inocencio F, Selimovic M, Raimundo L. Evidence Based Effectiveness of Pit and Fissure Sealants Applied by Students and Paediatric Dentists After Five Years. Acta Stomatol Croat. 2008;42:218-228.

10. San Martin L, Castaño A, Bravo M, Tavares M, Niederman R, Oqumbodede EO. Dental sealant knowledge, opinion, values and practice of Spanish dentists. BMC Oral Health . 2013 Feb 8;13:12.

11. MeSH Browser [database on the Internet]. Ministry of Health. Epidemiological Surveillance System of Oral Pathologies 10 years monitoring the oral health of Mexicans. 2018. Available from: http://www.epidemiologia.salud.gob.mx/doctos/infoepid/bol_ sivepab/SIVEPAB 10moaniv.pdf accessed January 232019.

12. MeSH Browser [database on the Internet]. 3M ESPE. Clinpro Sellador Nueva tecnología del cambio de color. 2018. [cited August 20 2018]Available from: http://multimedia.3m.com/mws/ media/1405590/3mtm-espetm-clinprotm-sealant.pdf

13. MeSH Browser [database on the Internet]. Shofu. BeautiSealant Fluoride Realasing Pit \& Fissure Sealant System. [cited August 20
2018]Available from: https://www.shofu.com/shofu_images/ Literature/beautisealant\%20brochure.pdf

14. Kantovitz KR, Moreira KM, Pascon FM, Nociti FH Jr, Machado Tabchoury CP, Puppin-Rontani RM. Penetration of Filled and Unfilled Resin Sealants on Different Enamel substrates. Pediatr Dent. 2016 Nov 15;38(7):472-476

15. Bhushan U, Goswami M. Evaluation of retention of pit and fissure sealants placed with and without air abrasion pretreatment in 6-8 year old children - An in vivo study. J Clin Exp Dent. 2017 Feb 1;9(2):e211-e217.

16. Paryab M. Sealant microleakage after using nano-filled bonding agents on saliva-contaminated enamel. J Dent (Tehran). 2013 May;10(3):227-32.

17. Erdemir U, Sancakli HS, Yaman BC, Ozel S, Yucel T, Yıldız E. Clinical comparison of a flowable composite and fissure sealant: a 24-month split-mouth, randomized, and controlled study. J Dent. 2014 Feb;42(2):149-57.

18. Griffin SO, Naavaal S, Scherrer C, Patel M, Chattopadhyay S; Community Preventive Services Task Force. Evaluation of School-Based Dental Sealant Programs: An Updated Community Guide Systematic Economic Review. Am J Prev Med. 2017 Mar;52(3):407-415.

19. Williams R, Rogo EJ, Gurenlian JR, Portillo KM. An evaluation of a school-based dental sealant programme. Int J Dent Hyg. 2018 May;16(2):e65-e72.

20. Guedes RS, Piovesan C, Ardenghi TM, Emmanuelli B, Braga MM, Mendes FM. Presence of Initial Caries Lesions as a Risk Factor for Caries in Preschool Children: A Cohort Study. Caries Res. 2018;52(1-2):32-41.

21. Vallejos-Sánchez AA, Medina-Solís CE, Casanova-Rosado JF, Maupomé G, Minaya-Sánchez M, Pérez-Olivares S. Caries increment in the permanent dentition of Mexican children in relation to prior caries experience on permanent and primary dentitions. J Dent. 2006 Oct;34(9):709-15.

22. Ikemura K, Tay FR, Endo T, Pashley DH. A review of chemical-approach and ultramorphological studies on the development of fluoride-releasing dental adhesives comprising new pre-reacted glass ionomer (PRG) fillers. Dent Mater J. 2008 May;27(3):315-39.

23. Dionysopoulos D, Sfeikos T, Tolidis K. Fluoride release and recharging ability of new dental sealants. Eur Arch Paediatr Dent. 2016 Feb;17(1):45-51.

24. Ushimura S, Nakamura K, Matsuda Y, Minamikawa H, Abe S, Yawaka Y. Assessment of the inhibitory effects of fissure sealants on the demineralization of primary teeth using an automatic $\mathrm{pH}$ cycling system. Dent Mater J. 2016;35(2):316-24.

25. Mickenautsch S, Yengopal V. Caries-Preventive Effect of High-Viscosity Glass lonomer and Resin-Based Fissure Sealants on Permanent Teeth: A Systematic Review of Clinical Trials. PLoS One. 2016 Jan 22;11(1):e0146512.

26. Deery C, Fyffe HE, Nugent ZI, Nuttall NM, Pitts NB. A proposed method for assessing the quality of sealants--the CCC Sealant Evaluation System. Community Dent Oral Epidemiol. 2001 Apr;29(2):83-91. 
27. Tianviwat S, Chongsuvivatwong V, Sirisakulveroj B. Loss of sealant retention and subsequent caries development. Community Dent Health. 2008 Dec;25(4):216-20.

28. Horuztepe SA, Ergin E, Onen A, Gürgan S. Comparison of Resin Infiltration Technique with Conventional Preventive Applications on Occlusal Fissures: EDS and SEM Analyses. Acta Stomatol Croat. 2020;54(4):382-391.
29. Fernández-Barrera MA, Lara-Carrillo E, Scougall-Vilchis RJ, Pontigo-Loyola AP, Ávila-Burgos L, Casanova-Rosado JF, et al. Study protocol of the cost-effectiveness comparison of two preventive methods in the incidence of caries: a randomized, controlled clinical trial. Medicine (Baltimore). 2019 Jul;98(30):e16634.

30. Garcia IM, Rodrigues SB, Leitune VCB, Collares FM. Antibacterial, chemical and physical properties of sealants with polyhexamethylene guanidine hydrochloride. Braz Oral Res. 2019 Mar 18;33:e019. 